infectious or malignant aetiology, although the histological findings are typically non-specific for a diagnosis of lupusrelated pericarditis. Pericardectomy is likely to be required for definitive treatment.

\section{PS10:185 NODULAR LOCALISED CUTANEOUS AMYLOIDOSIS IN A PATIENT WITH SYSTEMIC LUPUS ERYTHEMATOSUS}

${ }^{1,2} \mathrm{M}$ Deac Badarinza, ${ }^{1,2,3} \mathrm{~S}$ Rednic, ${ }^{1,2,3} \mathrm{C}$ Pamfil, ${ }^{2,4} \mathrm{C}$ Baican, ${ }^{5} \mathrm{~A}$ vasilache, ${ }^{1,2,3} \mathrm{I}$ Filipescu, II Muresan, 'D Balint, 1,3 L Damian. 'Rheumatology, Emergency Clinical County Hospital, Cluj-Napoca, Romania; ${ }^{2}$ Iuliu Hatieganu University of Medicine and Pharmacy, Cluj-Napoca, Romania; ${ }^{3}$ Rheumatology, Centre for Rare Musculoskeletal Autoimmune and Autoinflammatory Diseases, Cluj-Napoca, Romania; ${ }^{4}$ Dermatology, Emergency Clinical County Hospital, Cluj-Napoca, Romania; ${ }^{5}$ Hematology, Oncology Institute, Cluj-Napoca, Romania

\subsection{6/lupus-2018-abstract.226}

Introduction Nodular localised amyloidosis is a rare subtype of cutaneous amyloidosis, associated with various connective tissue diseases, mostly Sjogren's syndrome. Progression to systemic amyloidosis was described in 7\%-50\% cases. Amyloid deposition was also noted in hypertrofic lupus lesions.

Purpose To report a case of systemic lupus erythematosus (SLE) presenting with nodular localised cutaneous amyloidosis, followed up for 17 years.

Methods A 55 year patient was addressed to our tertiary unit with pain and swollen in both hands and multiple soft nodular lesions pink to brown on the chest and back.

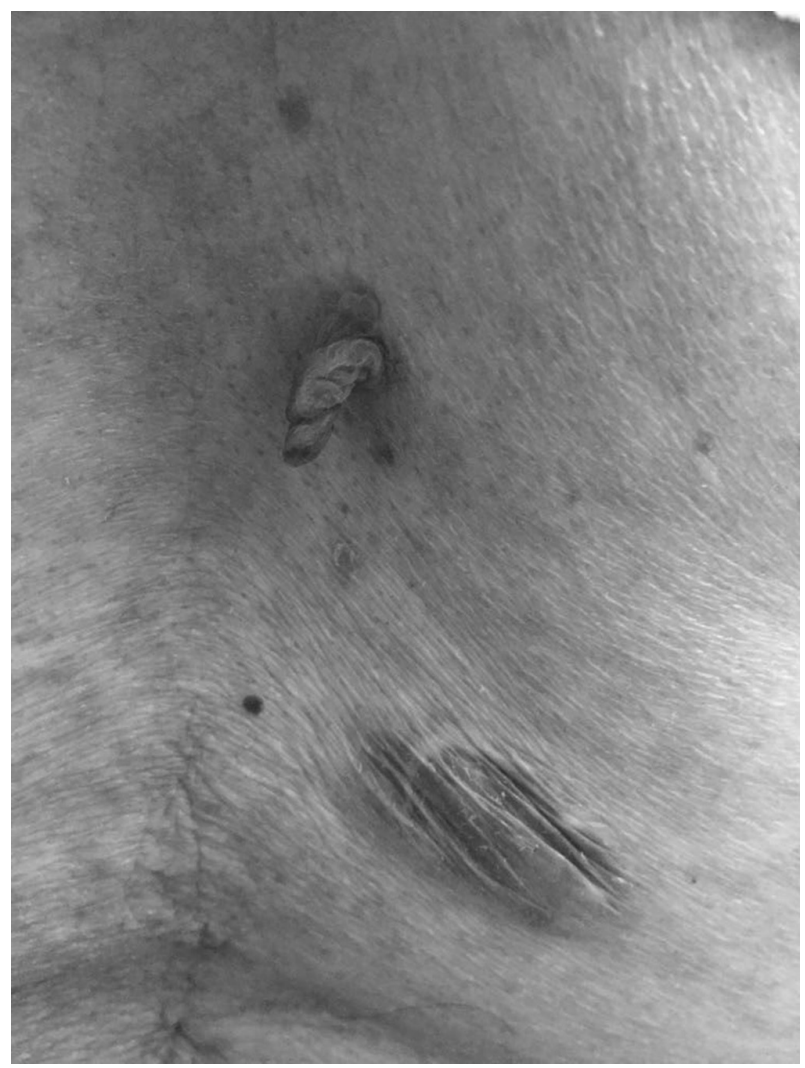

Abstract PS10:185 Figure 1

Results Clinical examination and further investigations revealed inflammatory hand arthritis and polyserositis including pericarditis and pleural effusion. Laboratory showed antinuclear antibodies with low anti-dsDNA titer, positive anti-Ro antibodies, positive rheumatoid factor, C3 and C4 consumption. She had negative anti -cyclic citrullinated peptide antibodies and anti-LA antibodies, no sicca symptoms and no ultrasound modification of the salivary glands. The skin histopathology with Congo red staining revealed amyloid deposition in the dermis. A screening for multiple myeloma, including bone marrow biopsy, was negative. She was treated with hydroxychloroquine, and over the time with methotrexate, azathioprine (with loss of tolerance), acitretin (with no significant skin improvement), and topical glucocorticorticoids. New lesions appeared mostly upon cessation of SLE therapy, on traumatised areas and sun exposure, but were quite stable during sustained systemic therapy, suggesting some relation to disease activity. She developed new-onset cryoglobulinemia with increasing anti- Ro titers and rheumatoid factor, but has still normal immunoglobulins, complement fractions and $\mathrm{LDH}$ and no light chains on immunelectrophoresis.

Conclusions Nodular localised amyloidosis is rare in SLE. The lesions evolve slowly, are minimally influenced by systemic therapy, but a close monitoring for systemic amyloidosis or plasma cell dyscrasia is required even in longstanding cases.

\section{PS10:187 CAUSES OF DEATH IN CHILDHOOD-ONSET SYSTEMIC LUPUS ERYTHEMATOSUS IN A TERTIARY CARE CENTRE, SOUTHERN THAILAND}

P Vachvanichsanong, E McNeil, P Dissaneewate. Prince of Songkla University, Hat Yai, Thailand

\subsection{6/lupus-2018-abstract.227}

Introduction The mortality rate of childhood-onset systemic lupus erythematosus (cSLE) remains high due to the severity of the disease and it complications. The cause of death varies widely depending on the major organs involved and therapy received.

Objective To evaluate the causes of death of cSLE.

Methods The medical records of children aged $<18$ years who had been diagnosed with SLE following American Rheumatism Association criteria from 1985 - 2016 in the Division of Nephrology, Department of Paediatrics, Prince of Songkla University, Thailand, were reviewed.

Results There were 331 patients, 272 girls and 59 boys, of whom $77(23.3 \%)$ died, 28.6\% within the first year after diagnosis. Only 29 medical records were available for evaluation of cause of death. Of these, there were 7 boys and 22 girls with a mean age at presentation of $11.0 \pm 3.0$ years. The mean follow-up duration was $4.6 \pm 3.7$ (range 0.2-12.6) years. The major cause of death was sepsis (13) followed by acute respiratory distress syndrome (ARDS) (6), severe heart condition (3), acute kidney injury (AKI) (2), chronic kidney disease (CKD) (2) and intracranial haemorrhage (1). Conditions at the time of death were sepsis (25), pneumonia (16), acute kidney injury (15), bleeding disorders (11), neurological complications (10), ARDS (10), CKD (4), AKI on top of CKD (3).

Conclusion The cause of death in cSLE is usually multi-factorial and it is difficult to assign a single dominant cause. Sepsis remains the most common cause of death. In the long-term, end-stage renal disease emerges as an important cause of death in RRT limited institutions. 


\section{PS10:189 NEUROPSYCHIATRIC LUPUS. A SEVERE MANIFESTATION OF SYSTEMIC LUPUS ERYTHEMATOSUS}

P Athanassiou, C Katsavouni, M Gatsiou, A Tzanavari, C Gerodimos. Department of Rheumatology, St. Paul's Hospital, Thessaloniki, Greece

\subsection{6/lupus-2018-abstract.228}

Purpose Nervous system involvement in systemic lupus erythematosus (SLE) is a grave manifestation of the disease affecting health, quality of life and disease outcome. It is one of the most complex manifestations of SLE and may affect the central, peripheral and autonomous nervous system. Complex interrelated pathogenetic mechanisms are involved in disease pathogenesis. The aim of the study was to describe a patient with neuropsychiatric lupus.

Methods A patient, female aged 50 years presented with SLE with a duration of 20 years. The diagnosis of the disease was made when she presented with intense fatigue, hair loss, a light sensitive rash, arthralgias and positive antinuclear and anti-dsDNA antibodies. In the course of the disease the patient developed CNS involvement with epileptic convulsions, permanent dysarthria and delusions. A brain MRI scan was without specific alterations, however an EEG performed was abnormal and a brain single-photon emission CT (SPECT) revealed decreased perfusion of both frontal and parietal lobes. The patient developed musculoskeletal manifestations at many stages of the disease. At diagnosis the patient had arthralgias of both wrists and knees. The patient presented with a flare with fatigue, mouth ulcers, convulsions, decreased ability to concentrate, intense delusions and dysarthria. At disease flare, when neuropsychiatric symptoms evolved she had diffuse arthralgias.

Results Pulse methylprednisolone i.v. followed by pulse cyclophosphamide i.v. were administered in order to achieve remission. Disease stabilisation was induced by pulse cyclophosphamide at bimonthly intervals and orally administered prednisolone. When remission of the disease was induced by pulse methylprednisolone and cyclophosphamide the patient developed muscle weakness. At disease stabilisation with pulse cyclophosphamide at bimonthly intervals the patient developed arthritis of the hand joints. The disease is now in remission, corticosteroid doses having been significantly reduced.

Conclusions Neuropsychiatric lupus is a grave and complex manifestation of SLE. The disease may be accompanied by various manifestations and severely affects quality of life. Neuropsychiatric lupus should be aggressively treated in order to improve quality of life and disease outcome.

\section{PS10:190 UNUSUAL PRESENTATION OF LUPUS PROFUNDUS}

PD Rath, A Abrari, S Budhiraja. Max Super Speciality Hospital, New Delhi, India

10.1136/lupus-2018-abstract.229

A middle aged female presented with swelling of face and periorbital region for 4 weeks and fever 3 weeks diagnosed and treated as cellulitis elsewhere examination revealed periorbital puffiness and indurated lesions on face with complete distortion of facial features.
Labs-hb- $10.7 \mathrm{gm} / \mathrm{dl}$

Tlc-4500/cumm

Plt- $160 \times 10^{\wedge} 3 /$ microlitre

Sr creatinine- $0.8 \mathrm{mg} / \mathrm{dl}$

Lft- sgot $-87 \mathrm{u} / \mathrm{L}$, sgpt- $64 \mathrm{u} / \mathrm{l}$

Ana if- neg

Ena profile- neg

Apla-neg

La-neg

C3, C4 n

$\mathrm{Ct}$ neck with contrast-diffuse enlargement of $\mathrm{rt}$ parotid gland
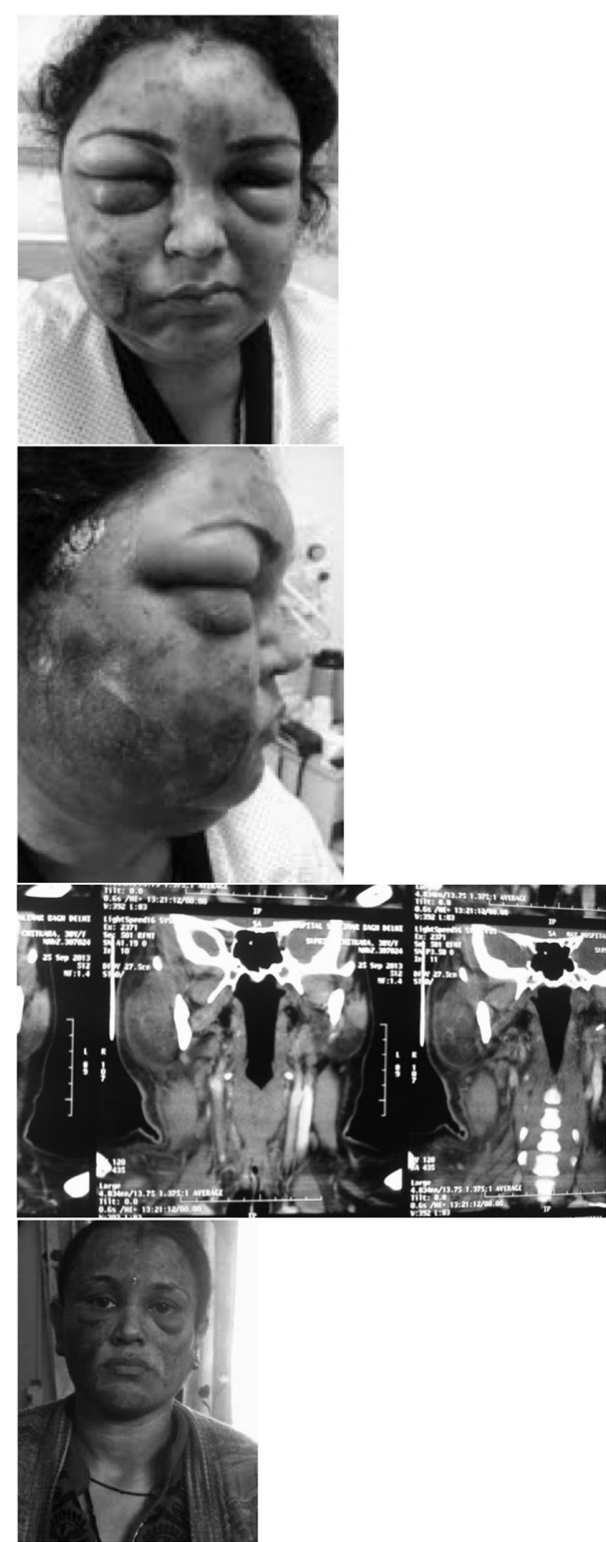

\section{Abstract PS10:190 Figure 1}

Swelling of $\mathrm{rt}$ masseter, buccinator sterno cleido mastoid

Gross oedema of overlying soft tissue of $\mathrm{rt}$ face multiple mildly enlarged $\ln$ rt sub mandibular upper and middle jugular erosion of alveolar cortex of rt post edge of maxilla.

Biopsy was done which showed -the subcut. Panniculus shows a lobular inflammatory infiltrate composed mainly of 